\title{
ZNF488 Enhances the Invasion and Tumorigenesis in Nasopharyngeal Carcinoma Via the Wnt Signaling Pathway Involving Epithelial Mesenchymal Transition
}

\section{Dan Zong, MD ${ }^{1}$ \\ Li Yin, $\mathrm{PhD}^{1}$ \\ Qian Zhong, PhD² \\ Wen-jie Guo, MD ${ }^{1}$ \\ Jian-hua $\mathrm{Xu}, \mathrm{MD}^{1}$ \\ Ning Jiang, $P h D^{1}$ \\ Zhi-rui Lin, $\mathrm{PhD}^{2}$ \\ Man-zhi Li, MD² \\ Ping Han, $\mathrm{PhD}^{3}$ \\ Lin $\mathbf{X u}, \mathrm{PhD}^{1}$ \\ Xia He, $\mathrm{PhD}^{1}$ \\ Mu-sheng Zeng, $\mathrm{PhD}^{2}$}

${ }^{1} J i a n g s u$ Cancer Hospital,

Nanjing Medical University,

Jiangsu Key Laboratory of Molecular and

Translational Cancer Research, Nanjing,

${ }^{2}$ State Key Laboratory of Oncology in South

China, Sun Yat-Sen University Cancer Center,

Guangzhou, ${ }^{3}$ Sun Yat-Sen Memorial Hospital,

Sun Yat-Sen University, Guangzhou, China

Correspondence: Xia He, $\mathrm{PhD}$

Jiangsu Cancer Hospital,

Nanjing Medical University, 42 Baiziting,

Nanjing 210009, China

Tel: 86-13601458518

Fax: 86-25-8328-3560

E-mail: hexia200302@tom.com

Co-Correspondence: Mu-sheng Zeng, PhD State Key Laboratory of Oncology in South China, Sun Yat-Sen University Cancer Center, 651 Dongfeng Road East, Guangzhou 510060, China

Tel: 86-20-8734-3191

Fax: 86-20-8734-3171

E-mail: zengmsh@mail.sysu.edu.cn

Received October 31, 2014

Accepted December 5, 2014

Published Online March 12, 2015

\section{Purpose}

The purpose of this study was to investigate the function of Zinc finger protein 488 (ZNF488) in nasopharyngeal carcinoma (NPC).

\section{Materials and Methods}

The endogenous expression of ZNF488 in NPC tissues, normal nasopharyngeal epithelium tissues and NPC cell lines were detected by quantitative reverse transcription polymerase chain reaction. ZNF488 over-expressing and knock-down NPC cell line models were established through retroviral vector pMSCV mediated over-expression and small interfering RNA (siRNA) mediated knock-down. The invasion and migration capacities were evaluated by wound healing and transwell invasion assays in ZNF488 over-expressing and control cell lines. Soft-agar colony formation and a xenograft experiment were performed to study tumorigenic ability in vitro and in vivo. Immunofluorescence and western blotting analysis were used to examine protein changes followed by ZNF488 over-expression. Microarray analysis was performed to explore gene expression profilings, while luciferase reporter assay to evaluate the transcriptive activity of Tcf/Lef.

\section{Results}

ZNF488 was over-expressed in NPC tissues compared with normal tissues, especially higher in 5-8F and S18, which are well-established high metastatic NPC clones. Functional studies indicate that over-expression of ZNF488 provokes invasion, whereas knock-down of ZNF488 alleviates invasive capability. Moreover, over-expression of ZNF488 promotes NPC tumor growth both in vitro and in vivo. Our data further show that over-expression of ZNF488 induces epithelial mesenchymal transition (EMT) by activating the WNT/ $\beta$-catenin signaling pathway.

\section{Conclusion}

Our data strongly suggest that ZNF488 acts as an oncogene, promoting invasion and tumorigenesis by activating the Wnt/ $\beta$-catenin pathway to induce EMT in NPC.

\section{Key words}

ZNF488, Nasopharyngeal carcinoma, Invasion, Carcinogenesis, Epithelial-mesenchymal transition, Wnt signaling pathway 


\section{Introduction}

Nasopharyngeal carcinoma (NPC) has a remarkably distinctive ethnic and geographic distribution. More than $80 \%$ of NPCs were reported in China, Southeast Asia, and several other Asian countries [1], with an annual incidence rate of approximately 20 per 100,000 people in endemic areas [2]. NPC is characterized by high invasion, migration, and proliferation abilities [3]. The standard treatment for NPC consists of concurrent chemo-radiotherapy with cisplatinbased regimens, generally followed by adjuvant chemotherapy [4]. Although advances are made in NPC clinical treatments, a significant proportion of NPC patients, especially those with stage III or IV, relapsed locoregionally and/or systemically. Thus, there is continuous calling for deeper understanding of the biological features in NPC.

Zinc finger protein represents one of the most abundant DNA-binding motifs in eukaryotic transcription factors, and the Cys2His2 ZNF motif is the most canonical type [5]. Recent studies closely link ZNF proteins to human cancer. For example, Zinc finger protein X-linked (ZFX) contributes to the stemness and pluripotent behavior of hepatocellular carcinoma [6]. ZNF703 protein is a novel oncoprotein regulating cell proliferation and migration [7]. ZNF217 protein may contribute to tumor progression in breast cancer by inducing epithelial mesenchymal transition (EMT) to enhance anti-apoptosis, invasion, and migration [8].

In an attempt to define the molecular mechanisms in the development and progression of NPC, cDNA microarray was utilized to examine differentially expressed genes between NPC tissues and normal nasopharyngeal tissues. Our results showed that Zinc finger protein 488 (ZNF488) was a major up-regulated gene in NPC tissues. ZNF488 is a nuclear, Kruppel-like zinc finger transcription factor [9]. The only known function of ZNF488 is the regulation of oligodendrocyte differentiation. ZNF488 is an oligodendrocytespecific transcriptional repressor that cooperates with the basic helix-loop-helix (bHLH) transcription factor Olig2 to promote oligodendrocyte differentiation and enhance myelin regeneration and repair in the central nervous system (CNS) following demyelinating diseases $[9,10]$. However, to our knowledge there is no comprehensive study of the effect of ZNF488 on human cancers. Therefore, the aim of our present study was to evaluate the expression and the possible function of ZNF488 in NPC.

\section{Materials and Methods}

\section{Tissue collection}

Thirty-four freshly frozen NPC specimens and 12 normal nasopharyngeal epithelium samples were retrieved from Sun Yat-Sen University Cancer Center (SYSUCC). None of the patients received any anti-tumor therapy prior to the biopsy sample collection. This study was approved by the Institutional Clinical Ethics Review Board of SYSUCC, and written informed consent was obtained from each patient.

\section{Cell culture}

Human NPC cells HNE1, CNE1, CNE2, HONE1, HK1, C666, SUNE1, SUNE2, CNE-2 subclones S18 and S26, and SUNE1 subclones 5-8F and 6-10B were maintained in RPMI1640 (Life Technologies, Carlsbad, CA) supplemented with $10 \%$ fetal bovine serum (FBS; Gibco Carlsbad, CA). NPEC2Bmi1 (N2-Bmi1), an immortalized nasopharyngeal epithelial cell, was established by transfection of oncogene Bmi-1 and maintained in keratinocyte medium (Invitrogen, Carlsbad, CA) in our laboratory [11]. NP69 was kindly provided by Dr George SW Tsao (University of Hong Kong, China) and cultured in Keratinocyte medium. The normal nasopharyngeal epithelial cells N01, N02, N03, N05, and N10P were obtained from normal nasopharyngeal mucosa using standard explant techniques [11], and routinely maintained in keratinocyte medium. The 293FT cell line was maintained in Dulbecco's modified Eagle medium (Invitrogen) supplemented with $10 \%$ FBS.

\section{RNA extraction, reverse transcription and quantitative polymerase chain reaction (RT-qPCR)}

Total RNA was isolated from tissues and cells using Trizol reagent (Invitrogen) and reverse-transcription using M-MLV reverse transcriptase (Promega, Madison, WI). Quantitative polymerase chain reaction (PCR) reactions using a Platinum SYBR Green qPCR SuperMix-UDG reagent (Invitrogen) were performed using Bio-RAD CFX96 real-time system (Bio-Rad, Hercules, CA). Reactions containing either no template or no reverse transcriptase were used as negative controls. Glyceraldehyde 3-phosphate dehydrogenase (GAPDH) was used as the normalization control. The primers used are listed in Table 1.

\section{Western blotting}

Total protein was extracted with sample buffer $(62.5$ $\mathrm{mmol} / \mathrm{L}$ Tris- $\mathrm{HCl}, \mathrm{pH} 6.8,2 \%$ sodium dodecyl sulfate [SDS], 
Table 1. Nucleotide sequences of the primers used for RTqPCR

\begin{tabular}{ll} 
Gene & \multicolumn{1}{c}{ Primers' sequence $^{c}$} \\
ZNF488 & F: 5'-CTTTCGCCTAACGTCCGA-3' \\
& R: 5'-GCTGTGAGAAGTCATGTGCC-3' \\
ABCG2 & F: 5'-TGCTCCAGCTTCTCCTTCTC-3' \\
& R: 5'-CATGTACTGGCGAAGAATATTTGGT-3' \\
NANOG & F: 5'-CCCAATACGACCAAATCCGTT-3' \\
& R: 5'-CTAAGAGGTGGCAGAAAAACA-3' \\
SOX2 & F: 5'-CACGTGATTCTTCCACAAGCC-3' \\
& R: 5'-CGAGTGGAAACTTTTGTCGGA-3' \\
GAPDH & F: 5'-CTCCTCCTGTTCGACAGTCAGC-3' \\
& R: 5'-CCCAATACGACCAAATCCGTT-3' \\
\hline
\end{tabular}

RT-qPCR, reverse transcription and quantitative polymerase chain reaction.

$10 \%$ glycerol, and $5 \% 2-\beta$-mercaptoethanol), and its concentration was quantified using the Pierce BCA Protein Assay Kit (Thermo, Massachusetts, MA). Total protein was subsequently separated on 6\%-12\% SDS-polyacrylamide gel electrophoresis gels and transferred onto polyvinylidene fluoride membranes (Millipore, Bedford, MA). The membranes were blocked with 5\% skim milk and incubated with primary antibodies, followed by incubation with anti-mouse or rabbit IgG secondary antibodies. Bands were detected by enhanced chemiluminescence, and GAPDH or $\alpha$-tubulin served as the loading control. Anti-ZNF488 was purchased from Abcam, Cambridge, UK; anti-ZO1, anti-E-cadherin, and anti- $\alpha$ catenin from BD Biosciences, Franklin Lakes, NJ; anti- $\alpha-$ tubulin, anti-Slug, anti-Snail, anti-phospho-GSK3 $\beta$ Ser9, and anti-GSK3 $\beta$ from Cell Signaling Technology, Boston, MA. Anti-vimentin (Novagen, South Africa), anti-N-cadherin (Epitomics, Burlingame, CA) and anti- $\beta$-catenin (Santa Cruz, Shanghai, China) were purchased.

\section{Plasmids and generation of stably transfected cell line}

ZNF488 cDNA was amplified by PCR and inserted into pcDNA3.1 and pMSCV vectors. The primers of ZNF488: BamHI-sense: 5'-CGGGATCCATGCCAGAGTGGCCAC-

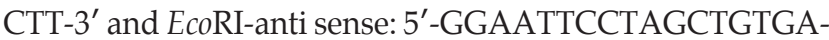
GAAGTCATGTGCC-3'. The vector or pMSCV-ZNF488 plasmid was transfected into 293FT cells along with the retroviral packaging vector PIK. After transfection, the supernatants were harvested and used to infect HNE1 and CNE1, and the stably transfected cells were selected with puromycin and validated by western blotting analysis.

\section{Small interfering RNA transfection}

Small interfering RNA (siRNA) transfections were performed using Lipofectamine RNAiMAX (Invitrogen) according to manufacturer's instructions. ZNF488 siRNAs were synthesised by RiboBio (Guangzhou, China). The targeting sequences were as follows: si-ZNF488-1, 5'-GCGCCTTTAGCAAACCAAC-3'; si-ZNF488-2, 5'-GCAAAGTGCAACCTGTCCT-3'; and si-ZNF488-3, 5'-CCTGGTCTTTCACATGCG A-3'.

\section{Wound healing assay}

Cells were seeded and allowed to reach 70\%-80\% confluence, then starved for 36 hours. The cell monolayers were then wounded with a sterile plastic tip and cultured in serum-free medium. Cell migration was monitored every 12 hours using microscopy (Nikon, Tokyo, Japan).

\section{Transwell invasion assay}

Cells were plated on top of a thick layer of Matrigel in transwell chambers (BD Biosciences). After culturing for 18 hours, non-invasive cells on the upper surface of filters were removed completely. Invasive cells adhered to the lower surface of filter were rinsed with phosphate-buffered saline (PBS), fixed with methanol, stained with $0.05 \%$ crystal violet and counted.

\section{Soft-agar colony formation assay}

Plates were coated with a layer of $1 \%$ agar in $20 \%$ FBS medium. Cells were prepared in $0.66 \%$ agar in $10 \%$ FBS medium and seeded. Plates were incubated until colonies formed. Colonies were counted and photographed with a microscope (Nikon).

\section{Xenograft experiment}

Nude mice were purchased from the Guangdong Laboratory Animal Co. Ltd. (Guangzhou, China) and maintained in micro-isolator cages. All animals were used in accordance with institutional guidelines, and the Committee for Animal Care and Use approved the experiments. Tumor cells were suspended in $100 \mathrm{~mL}$ RPMI-1640 FBS-free medium with 25\% Matrigel (BD Biosciences) and inoculated subcutaneously into the left flanks of 4-week-old nude mice. The mice were monitored daily for palpable tumor formation, and tumors were measured, weighed and photographed. 
A

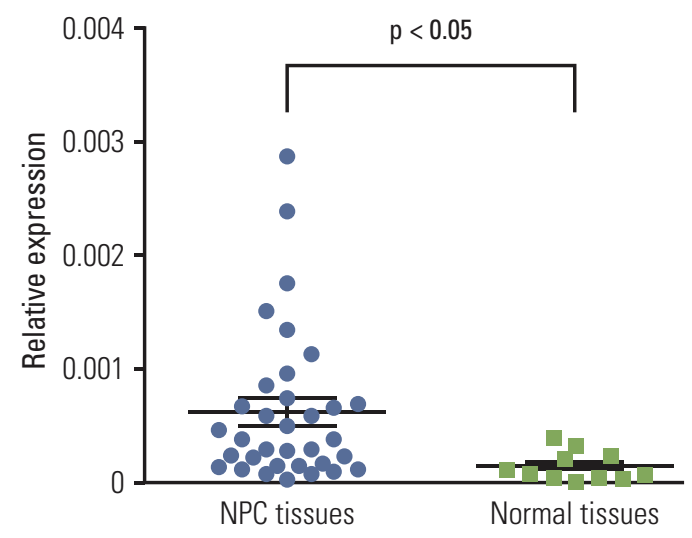

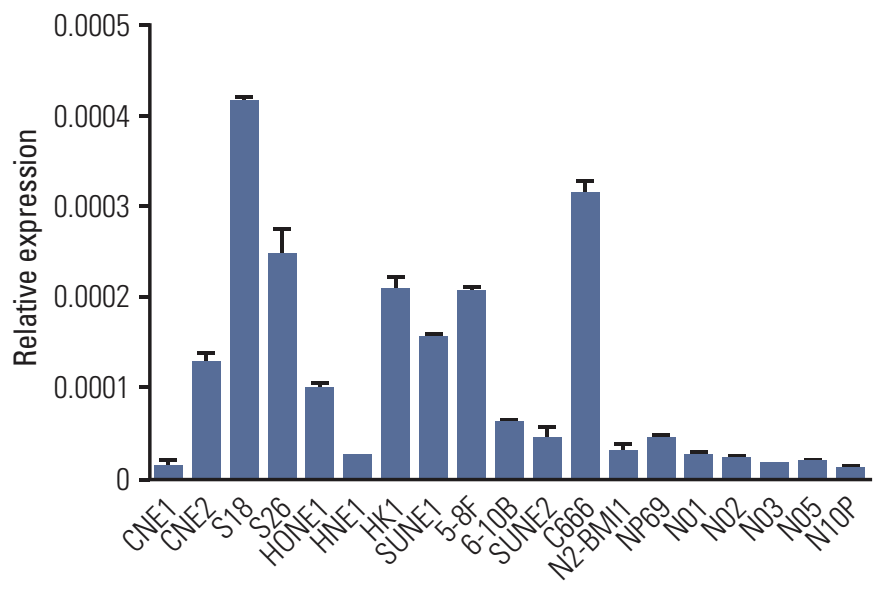
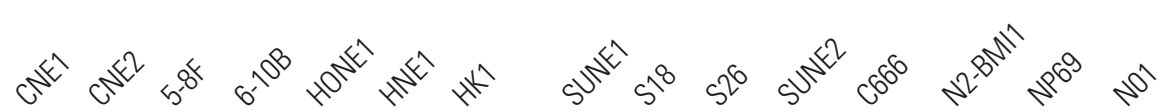

C
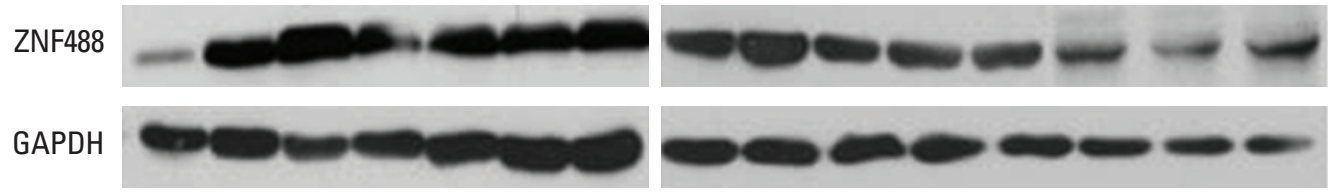

Fig. 1. Zinc finger protein 488 (ZNF488) is highly expressed in nasopharyngeal carcinoma (NPC). (A) Expression levels of ZNF488 mRNA in NPC tissues and normal nasopharyngeal epithelial tissues. Glyceraldehyde 3-phosphate dehydrogenase (GAPDH) was used as the endogenous control. (B, C) Expression levels of ZNF488 mRNA (B) or protein (C) in a series of NPC cell lines and immortalized or normal nasopharyngeal epithelial cells. Data are presented as the mean \pm standard deviation, and p-values were calculated with Student's t test.

\section{Immunofluorescence staining}

Cells were plated on multiwall coverslips to $80 \%$ confluence, washed three times in PBS, and fixed in freshly prepared $4 \%$ polyphosphate formaldehyde for 10 minutes. The cells were incubated in PBS three times: 10 minutes with PBS containing 0.5\% Triton X-100 (selected); 2 times with PBS,5 minutes per time; and blocked in $5 \%$ bovine serum albumin for 30 minutes. Cells were incubated with primary antibodies overnight at $4^{\circ} \mathrm{C}$. After washing three times with PBS, slides were incubated with Alexa Fluor* 488 or 594 goat anti-mouse or anti-rabbit IgG antibodies (Invitrogen) for 1 hour. Slides were counterstained with 4-6-diamidino-2-phenylindole (DAPI, Sigma, St. Louis, MO) to visualize nuclei, followed by laser scanning confocal microscope (IX81, Olympus, Tokyo, Japan).

\section{Microarray analysis}

RNA was extracted from the paired stable cell lines HNE1-
Vector, HNE1-ZNF488, CNE1-Vector, and CNE1-ZNF488 using TriPure Isolation Reagent (Roche, Vilvoorde, Belgium) and the Qiagen RNAeasy Mini Kit (Qiagen, Venlo, The Netherlands). RNA was amplified and labelled using the Affymetrix GeneChip 3'IVT Express Kit (Affymetrix, Sacramento, CA); and the cDNA was then hybridised to an Affymetrix GeneChip HG-U133 Plus 2.0, stained using an Affymetrix GeneChip Fluidics Station 450 and scanned using the GeneChip Scanner 3000. Quality control was carried out using the Affymetrix GCOS software. Genes up- and downregulated by 1.5- and 2-fold, respectively, between the paired stable cell lines were analyzed.

\section{Transfection and luciferase reporter assay}

Transfection was performed using Lipofectamine 2000 (Invitrogen) according to manufacturer's instructions. Cells were co-transfected with $200 \mathrm{ng}$ pcDNA3.1 or pcDNA3.1ZNF488, 25 ng pTK-RL (Renilla, Madison, IN) and 300 ng TopFlash/FopFlash plasmid (Millipore). Cells were har- 
A
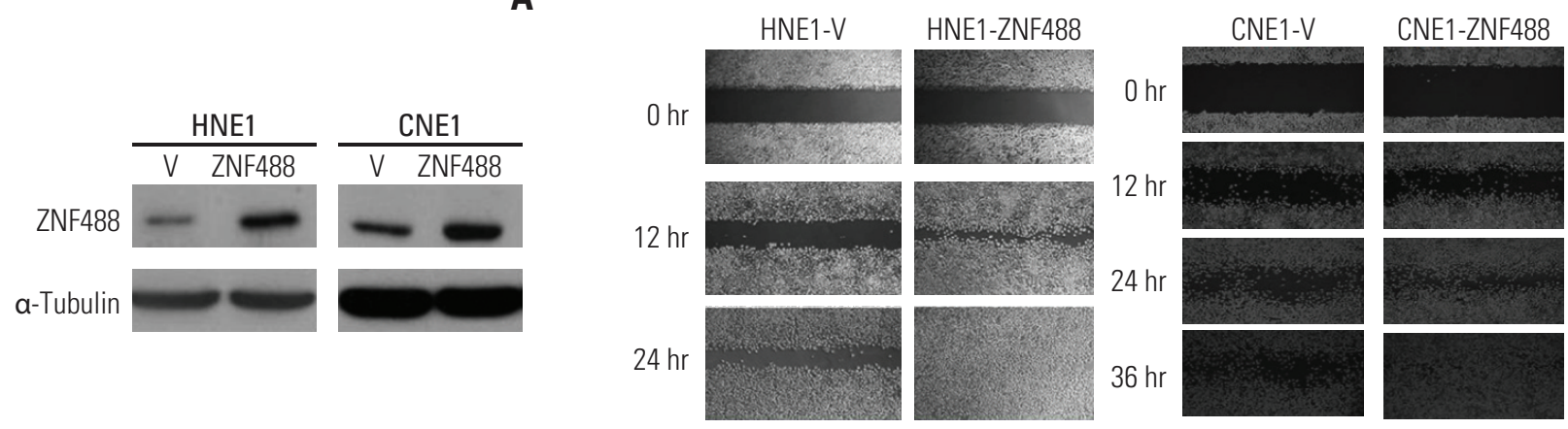

C
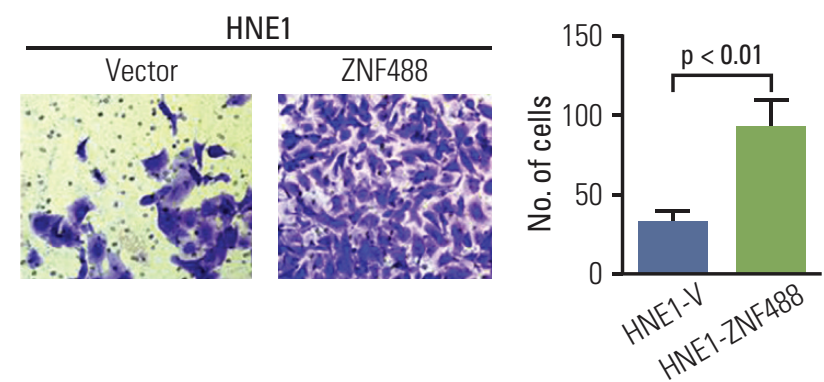<smiles>CC(C)[Hg][Mg][Mg]</smiles>
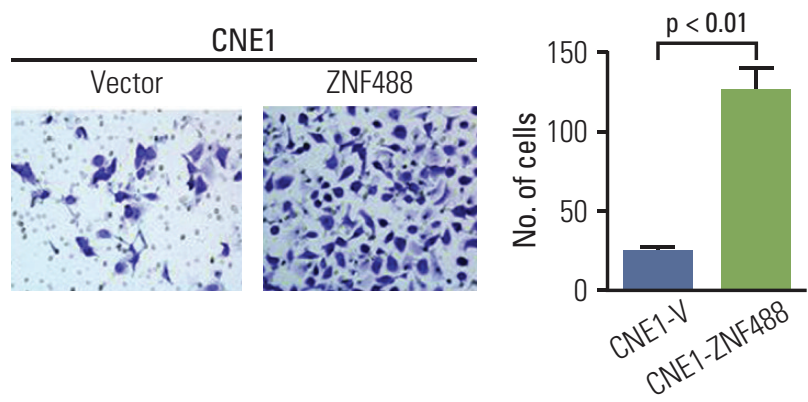

GAPDH
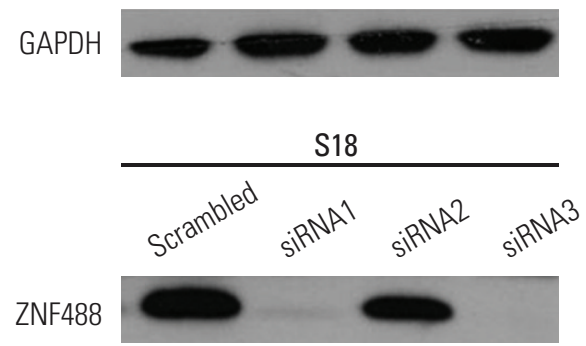

GAPDH

. 


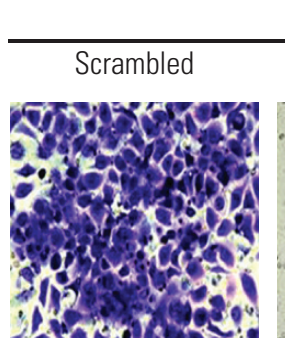

\section{5-8F}

SiRNA1

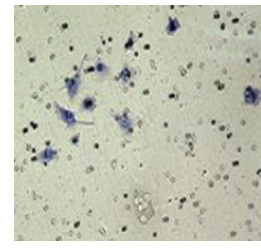

S18

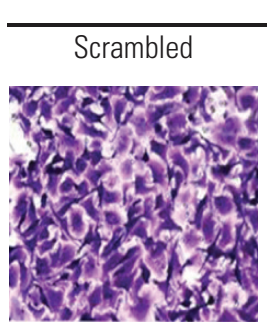

SiRNA1

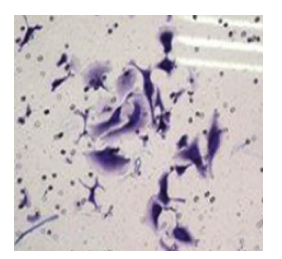

SiRNA3

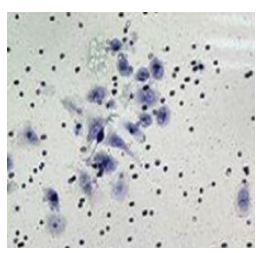

siRNA3

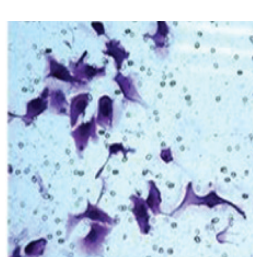

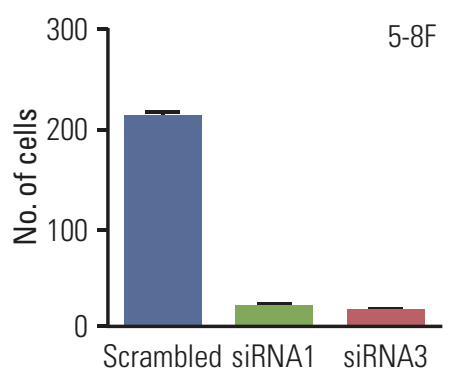

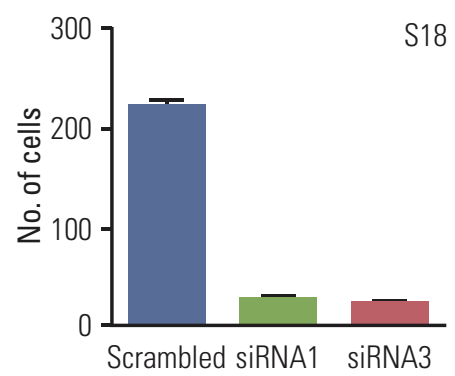

Fig. 2. (Continued from the previous page) (E) 5-8F and S18 cells were transfected with two individual ZNF488 siRNAs or a scrambled control followed by transwell invasion assays. Data are presented as the mean \pm standard deviation, and chance was ruled out with Student's t test.

\section{Results}

\section{ZNF488 is highly expressed in NPC}

Based on our cDNA microarray results, we found that ZNF488 was identified as a major up-regulated gene in NPC tissue compared with normal nasopharyngeal epithelial tissue (data not shown). To further validate our finding, we detected ZNF488 mRNA expression in 34 freshly frozen primary NPC tissues and 12 normal nasopharyngeal epithelial tissues. Our results showed that ZNF488 mRNA levels were considerably higher in NPC tissues $(\mathrm{p}<0.05)$ (Fig. 1A). Furthermore, quantitative reverse transcription polymerase chain reaction (RT-PCR) and western blotting analyses were used to determine the expression levels of ZNF488 mRNA and protein in NPC cell lines, two immortalized nasopharyngeal epithelial cells (N2-bmi1 and NP69) and five primary normal nasopharyngeal epithelial cells ( $\mathrm{N} 01, \mathrm{~N} 02, \mathrm{~N} 03, \mathrm{~N} 05$, and N10P). Higher expression of ZNF488 mRNA was detected in eight of 12 NPC cells compared with immortalized and normal nasopharyngeal epithelial cells (Fig. 1B). Consistent with our quantitative RT-PCR results, ZNF488 protein was also elevated in NPC cell lines compared with normal nasopharyngeal epithelial cells (Fig. 1C). These results suggest that ZNF488 is up-regulated in NPC.

\section{Over-expression of ZNF488 promotes invasion and migration in NPC cell lines}

To investigate the function of ZNF488 in NPC, we exogenously over-expressed ZNF488 in two NPC cells, HNE1 and CNE1. Exogenous expression of ZNF488 was confirmed by western blotting (Fig. 2A). Cells were then tested for wound healing assay. For this test, the cells were starved for 36 hours and cultured in serum-free medium, to exclude the interference attributable to the proliferative action. As shown in Fig. 2B, over-expression of ZNF488 strongly simulated cell migration. Similar results were confirmed by transwell invasion assays. Numbers of invasive cells were significantly higher in ZNF488 over-expressing cells ( $p<0.01)$ (Fig. 2C). To prove that endogenous ZNF488 regulates cell migration and invasion, we conducted siRNA-targeted ZNF488 knockdown in two high-metastatic cell lines, 5-8F and S18. Efficient knock-down of ZNF488 expression was confirmed by western blotting (Fig. 2D), with more efficient knock-down by siRNA 1 and siRNA 3. As expected, knock-down of ZNF488 expression significantly suppressed cell invasion $(\mathrm{p}<0.01)$ (Fig. 2E). In conclusion, these data suggest that ZNF488 promotes migration and invasion in NPC cells. 
A
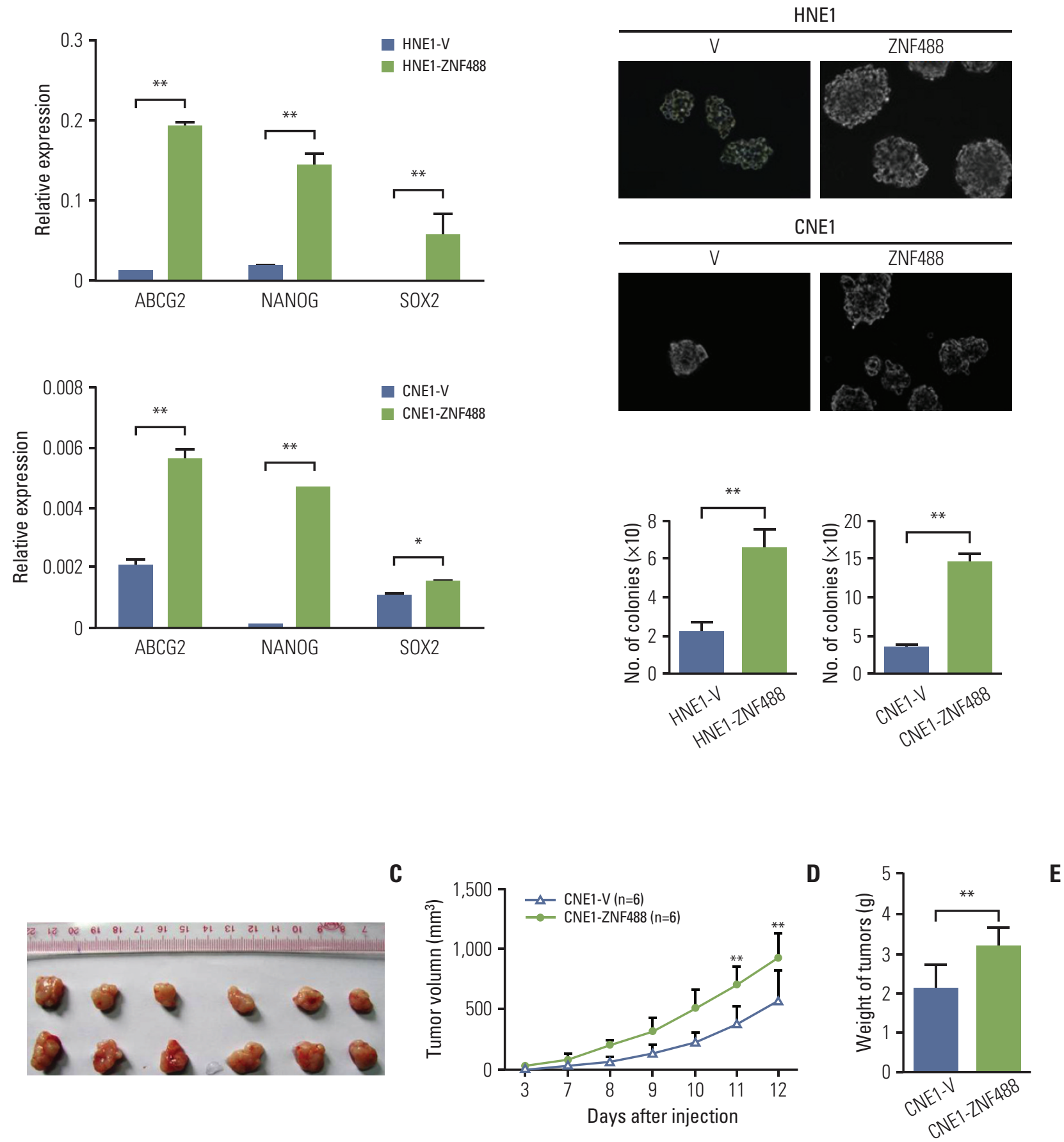

Fig. 3. Zinc finger protein 488 (ZNF488) induces cancer stem cell-like self-renewal properties to enhance tumorigenesis. (A) Expression of stem cell markers, ABCG2, NANOG, and SOX2 by reverse transcription and quantitative polymerase chain reaction analysis. (B) ZNF488-over-expressing and control cell lines were subjected to anchorage-independent colony formation in soft agar $(\times 20)$. (C) Representative picture of xenograft tumors in nude mice inoculated with CNE1 stably expressing ZNF488 or vector control. (D) Growth curves of tumor volumes in nude mice. (E) Statistical analysis of tumor weights. Data are presented as the mean \pm standard deviation, and $\mathrm{p}$-values were calculated with Student's t test. ${ }^{*} \mathrm{p}<0.05,{ }^{* *} \mathrm{p}<0.01$. 
A

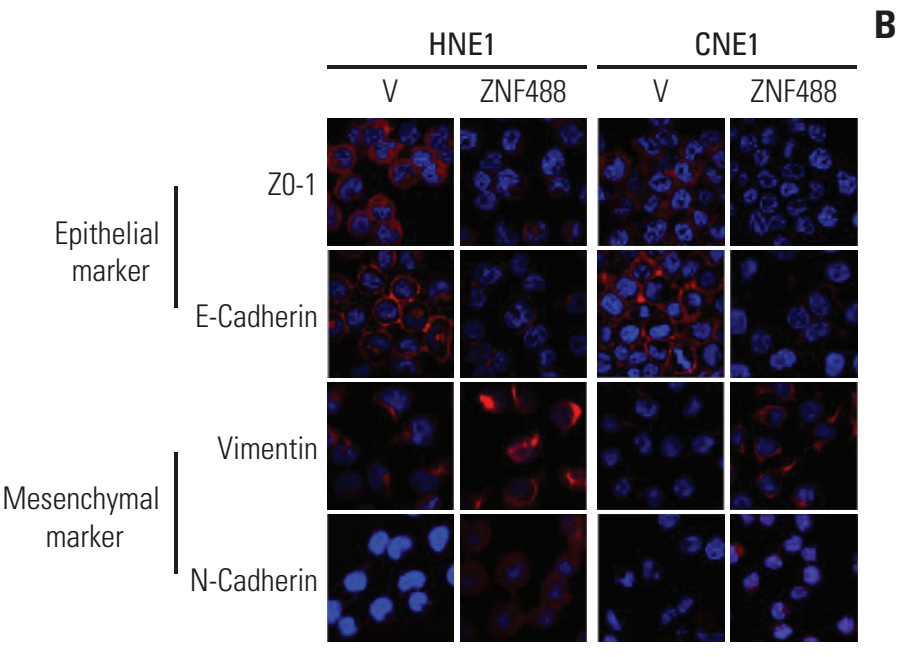

C
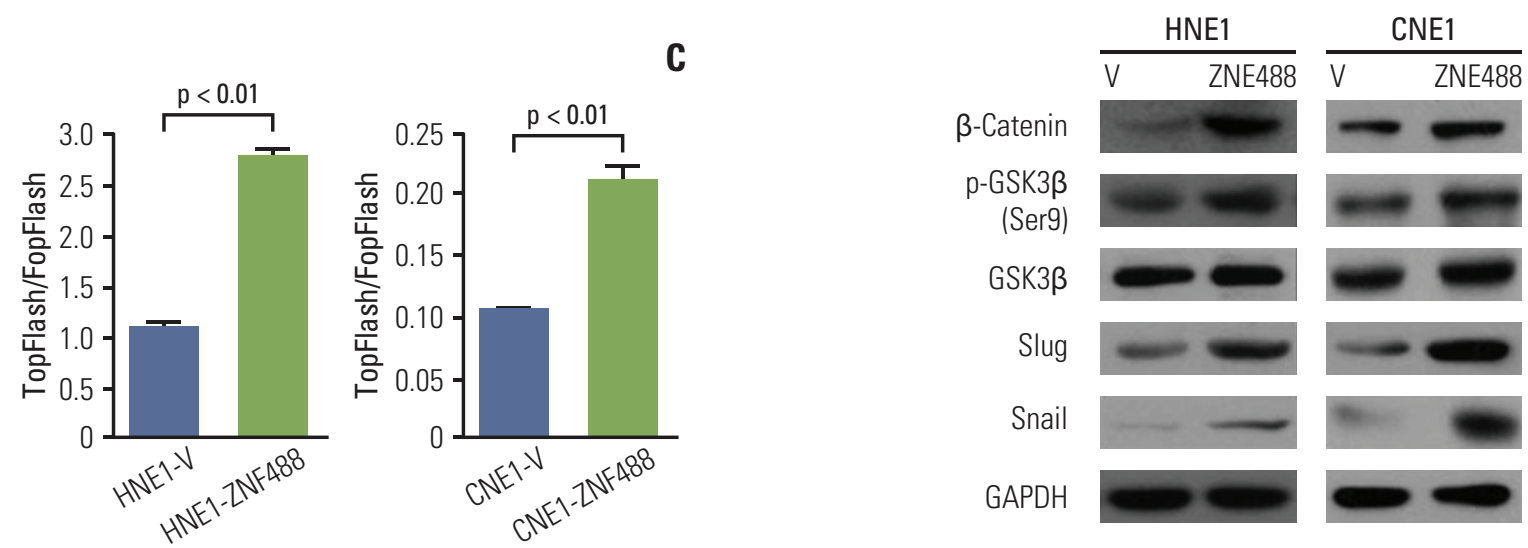

Fig. 4. Zinc finger protein 488 (ZNF488) activates the WNT/ $\beta$-catenin pathway to induce the epithelial mesenchymal transition (EMT) transcription factors Tcf / Lef which is capable of triggering EMT. (A) The protein level of E-cadherin, $\alpha$-catenin, vimentin, and $\mathrm{N}$-cadherin were detected by western blotting analysis. (B) Immunofluorescence staining to dectect the changes of E-cadherin, ZO-1, vimentin, and N-cadherin. (C) Luciferase reporter assays using TopFlash/FopFlash reporter plasmids to monitor the activity of Tcf/Lef transcription factor. (D) Western blotting analysis to detect the expression of $\beta$-catenin, phospho-GSK3 $\beta$ (Ser9), GSK3 $\beta$, Slug, and Snail. GAPDH, glyceraldehyde 3-phosphate dehydrogenase. Data are presented as the mean \pm standard deviation, and p-values were calculated with Student's $t$ test.

\section{ZNF488 induces cancer stem cell-like self-renewal prop- erties to enhance tumorigenesis}

We also studied the effects of ZNF488 on cancer stem cell property. Representative cancer stem cell markers, Nanog, ABCG2, and Sox2, were detected using RT-qPCR. Overexpression of ZNF488 markedly up-regulated the mRNA levels of Nanog, ABCG2, and Sox2 in both HNE1 and CNE1 stable cell lines (Fig. 3A). In addition, the tumor growth ability of cells on soft agar indicated the growth of cancer stem cell populations. We found that over-expression of ZNF488 significantly increased the ability of anchorage-independent growth in soft agar (Fig. 3B). To explore whether ZNF488 was required for NPC tumor growth in vivo, we conducted xenograft tumor model assays by subcutaneously injecting stably constructed ZNF488-over-expressing CNE1 or control CNE1 cells into the left flanks of six nude mice. Tumors were weighed and photographed (Fig. 3C) 12 days after NPC cells injection. Our data showed that the growth rate of tumors derived from ZNF488-over-expressing cells was significantly faster than control cells $(p<0.01)$ (Fig. 3D). Tumors formed in ZNF488-over-expressing group were much heavier than in the control group $(\mathrm{p}<0.01)$ (Fig. 3E). These data indicate that ZNF488 stimulates the generation of cancer 
Table 2. Pathway analysis of 1.5 folds or more common changed genes

\begin{tabular}{lll} 
Category & \multicolumn{1}{c}{ Term } & p-value \\
Kegg pathway & Tight junction & 0.007181 \\
Panther pathway & Wnt signaling pathway & 0.019056 \\
Panther pathway & B-cell activation & 0.034594 \\
\hline
\end{tabular}

stem cell-like properties, which might facilitate the tumorigenesis both in vitro and in vivo.

\section{ZNF488 activates the $\mathrm{Wnt} / \beta$-catenin pathway to induce the EMT transcription factor Tcf/Lef, which promotes the EMT in NPC}

EMT is involved in the invasive capacity of transformed epithelial cells [12]. We examined whether ZNF488 could induce EMT in NPCs. A key molecular feature in EMT is the down-regulation of E-cadherin, which is tightly controlled by Snail-related zinc-finger transcriptional repressors [13]. As shown in Fig. 4A, the epithelial markers E-cadherin and $\alpha$-catenin were down-regulated, whereas mesenchymal markers vimentin and $\mathrm{N}$-cadherin were up-regulated compared with their controls. These observations were further verified by immunofluorescence staining (Fig. 4B), suggesting that over-expression of ZNF488 lead to an EMT phenotypic transition.

To gain mechanistic insights into ZNF488-driven tumor invasion and tumorigenesis, we performed microarray analysis in both HNE1 and CNE1 stable cell lines. Pathway analysis using genes showing 1.5 -folds or more common changes and the Database for Annotation, Visualization and Integrated Discovery (DAVID) v6.7 revealed that the inflammatory, tight junction, and Wnt signaling pathway differed significantly. Among these, genes in the Wnt signaling pathway, including cadherin 7, PCDHB17, LEF1, PCDHA1, PLCB1, NFATC2, PRKCB, and $\beta$-catenin, were significantly different $(p<0.05)$ (Table 2$)$. The WNT signaling pathway, implicated in EMT, promotes tumor initiation and progression and contributes to the acquisition of self-renewal and metastatic phenotypes $[14,15]$. Based on the microarray results, we sought to determine whether the $\mathrm{Wnt} / \beta$-catenin pathway is involved in the ZNF488-induced EMT. We evaluated the activity of Tcf/Lef transcription factor, involved in the Wnt signaling pathway, by luciferase reporter assays. As shown in Fig. 4C, over-expression of ZNF488 enhanced $\mathrm{Tcf} /$ Lef transcriptional activity in both cell lines. The protein levels of $\beta$-catenin and $p$-GSK3 $\beta$ were up-regulated with the over-expression of ZNF488 (Fig. 4D). We found that overexpression of ZNF488 also up-regulated the expression of
EMT transcription factors Snail and Slug (Fig. 4D). Thus, the activating of Wnt / $\beta$-catenin signaling pathway may play a role in ZNF488 promoted EMT transition.

\section{Discussion}

NPC is a disease with remarkable racial and geographic distribution. Virological, genetic and environmental factors are involved in the etiology of NPC, although the molecular basis of NPC is not well understood. ZNF488 is a zinc finger protein of ZNF family, which is highly conserved in vertebrates. It is required for oligodendrocyte precursor formation and differentiation during embryonic development [10], and has important implications in CNS myelin regeneration and repair after demyelinating diseases. However, the role of ZNF488 in cancer, especially in NPC is poorly understood.

In this study, our findings suggest an important role of ZNF488 in NPC. We showed that ZNF488 expression was significantly higher in NPC tissues compared with normal epithelial tissues, consistent with our previous results of transcriptome sequencing. Importantly, ZNF488 was overexpressed at both mRNA and protein levels in NPC cell lines. It is interesting to note that two subclones derived from parental CNE2, S18 and S26, S18 with higher percentages of spindle-shaped cells, indicated high invasive and metastatic ability [16]. SUNE1-derived subclones 5-8F and 6-10B, 5-8F were highly tumorigenic and metastatic [17]. Intriguingly, both highly metastatic S18 and 5-8F expressed higher levels of ZNF488 than their counterparts. Based on these results, we hypothesized that ZNF488 is implicated in the invasion of NPC.

We further provided evidence showing that over-expression of ZNF488 remarkably stimulated migration and invasion, while knock-down of ZNF488 alleviated invasion in NPC cells. EMT, as a prerequisite for cancer invasion and metastasis, leads to the up-regulation of mesenchymal markers toward mesenchymal phenotype [18]. This transformation is associated with tumor invasiveness, metastasis, and resistance to chemotherapy. Our results showed that epithelial markers E-cadherin and $\alpha$-catenin were down-regulated, whereas the mesenchymal markers vimentin and $\mathrm{N}$-cadherin were up-regulated in ZNF488-over-expressing cell lines, indicating that over-expression of ZNF488 triggered an EMTlike phenotypic transition.

EMT is implicated in the successful formation of micrometastasis by conferring cancer stem cell properties to disseminated cancer cells [12]. Cancer stem cells appear to drive both tumorigenesis and metastasis, while resistant to standard radiation and chemotherapy [19]. We found that 
EMT upon ZNF488 over-expression was accompanied by stem cell-like phenotype, as determined by increased expression of stem-cell markers Nanog, Sox2, and ABCG2 [20,21]. Furthermore, ZNF488 over-expressing cells possessed more tumorigenic capacity than control cells in vitro and in vivo. New therapeutic approaches have been explored to target this population. One strategy is to target critical signaling pathways for self-renewal and differentiation. Several candidate pathways have been identified including Notch, Wnt, and Hedgehog [22-24]. Thus, ZNF488-induced EMT enhances stem cell-like self-renewal properties that are crucial in tumorigenesis. Mechanistically, microarray analysis revealed that $\mathrm{Wnt} / \beta$-catenin signaling pathway might be involved in ZNF488-induced EMT. A number of cytokines and growth factors are induced during EMT, which amplifies the EMT program and promotes cell migration. These factors include the cytokines TGF- $\beta$, Wnt, Notch ligands, interleukin-like EMT-inducers [25]. One of the signals initiating EMT was the canonical Wnt pathway, whose stimulation triggered the translocation of oncoprotein $\beta$-catenin to nucleus, where it acted as coactivator of Tcf/Lef factor in transcriptional activation of target genes. We found that over-expression of ZNF488 activated the Wnt/ $\beta$-catenin signaling pathway to enhance Tcf/Lef transcriptional activity, thereby contributing to the EMT. Additionally, the activation of Wnt/ $\beta$-catenin pathway was often coupled with the expression of EMT regulators such as snail and slug. Snail and Slug regulate tight junction stability, gap junctional protein expression, desmosome disassembly and protease expression. Indeed, Snail and Slug are expressed in the response to EMT inducing stimuli including Wnt signaling [25]. As a result, the transcription factors Snail and Slug were elevated in ZNF488-over-expressing cell lines. Thus, we hypothesized that ZNF488 activates the Wnt/ $\beta$-catenin signaling pathway, leading to the activation of the Tcf/Lef transcription factor.

As ZNF488 is highly conserved, silico analysis revealed that ZNF488 contains a possible nuclear localization signal (NLS) sequence: a bipartite NLS between its two zinc-finger motifs that functions as a transcriptional repressor. This study, however, did not determine the exact binding site of
ZNF488 target genes. Regardless of the direct binding site, our substantial evidence reveals, for the first time, the importance of ZNF488 in invasion and tumorigenesis in NPC. It holds promise as a novel molecular target for NPC therapy and a potential biomarker for predicting patient prognosis.

\section{Conclusion}

In conclusion, the present study indicated that ZNF488 was over-expressed in NPC, and over-expression of ZNF488 could enhance the invasive and tumorigenic abilities in NPC. Our findings provide new insights into the molecular mechanisms involved in the regulation of NPC invasion and might provide novel therapeutic targets and strategies for the treatment of NPC patients.

\section{Conflicts of Interest}

Conflict of interest relevant to this article was not reported.

\section{Acknowledgments}

We thank Dr. George SW Tsao at University of Hong Kong for providing cell lines. We thank Tian-liang Xia for expert technical assistance. This study was partly supported by a grant from the Ministry of Science and Technology of China (2012CB967003 to M.-S. Z) and the National Natural Science Foundation grants of China (81230045, 81025014 to M.-S. Z), a National Natural Science Foundation grant of China (81372321 to L. X) and the Key Clinical Medicine Technology Foundation of Jiangsu province (BL2012030 to L. X).

\section{References}

1. Lee AW, Ng WT, Chan YH, Sze H, Chan C, Lam TH. The battle against nasopharyngeal cancer. Radiother Oncol. 2012;104: 272-8.

2. Zhang L, Zhao C, Ghimire B, Hong MH, Liu Q, Zhang Y, et al. The role of concurrent chemoradiotherapy in the treatment of locoregionally advanced nasopharyngeal carcinoma among endemic population: a meta-analysis of the phase III randomized trials. BMC Cancer. 2010;10:558.

3. Xu ZJ, Zheng RS, Zhang SW, Zou XN, Chen WQ. Nasopharyngeal carcinoma incidence and mortality in China in 2009. Chin J Cancer. 2013;32:453-60.

4. Razak AR, Siu LL, Liu FF, Ito E, O'Sullivan B, Chan K. 
Nasopharyngeal carcinoma: the next challenges. Eur J Cancer. 2010;46:1967-78.

5. Lupo A, Cesaro E, Montano G, Zurlo D, Izzo P, Costanzo P. KRAB-Zinc finger proteins: a repressor family displaying multiple biological functions. Curr Genomics. 2013;14:268-78.

6. Lai KP, Chen J, He M, Ching AK, Lau C, Lai PB, et al. Overexpression of ZFX confers self-renewal and chemoresistance properties in hepatocellular carcinoma. Int J Cancer. 2014;135: 1790-9.

7. Yang G, Ma F, Zhong M, Fang L, Peng Y, Xin X, et al. ZNF703 acts as an oncogene that promotes progression in gastric cancer. Oncol Rep. 2014;31:1877-82.

8. Vendrell JA, Thollet A, Nguyen NT, Ghayad SE, Vinot S, Bieche I, et al. ZNF217 is a marker of poor prognosis in breast cancer that drives epithelial-mesenchymal transition and invasion. Cancer Res. 2012;72:3593-606.

9. Wang SZ, Dulin J, Wu H, Hurlock E, Lee SE, Jansson K, et al. An oligodendrocyte-specific zinc-finger transcription regulator cooperates with Olig2 to promote oligodendrocyte differentiation. Development. 2006;133:3389-98.

10. Soundarapandian MM, Selvaraj V, Lo UG, Golub MS, Feldman DH, Pleasure DE, et al. Zfp488 promotes oligodendrocyte differentiation of neural progenitor cells in adult mice after demyelination. Sci Rep. 2011;1:2.

11. Song LB, Zeng MS, Liao WT, Zhang L, Mo HY, Liu WL, et al. Bmi-1 is a novel molecular marker of nasopharyngeal carcinoma progression and immortalizes primary human nasopharyngeal epithelial cells. Cancer Res. 2006;66:6225-32.

12. Mani SA, Guo W, Liao MJ, Eaton EN, Ayyanan A, Zhou AY, et al. The epithelial-mesenchymal transition generates cells with properties of stem cells. Cell. 2008;133:704-15.

13. Song LB, Li J, Liao WT, Feng Y, Yu CP, Hu LJ, et al. The polycomb group protein Bmi-1 represses the tumor suppressor PTEN and induces epithelial-mesenchymal transition in human nasopharyngeal epithelial cells. J Clin Invest. 2009; 119:3626-36.

14. Anastas JN, Moon RT. WNT signalling pathways as therapeutic targets in cancer. Nat Rev Cancer. 2013;13:11-26.

15. Yu M, Ting DT, Stott SL, Wittner BS, Ozsolak F, Paul S, et al. RNA sequencing of pancreatic circulating tumour cells implicates WNT signalling in metastasis. Nature. 2012;487:510-3.
16. Deng CC, Liang Y, Wu MS, Feng FT, Hu WR, Chen LZ, et al. Nigericin selectively targets cancer stem cells in nasopharyngeal carcinoma. Int J Biochem Cell Biol. 2013;45:1997-2006.

17. Liu X, Lv XB, Wang XP, Sang Y, Xu S, Hu K, et al. MiR-138 suppressed nasopharyngeal carcinoma growth and tumorigenesis by targeting the CCND1 oncogene. Cell Cycle. 2012;11: 2495-506.

18. Wendt MK, Schiemann BJ, Parvani JG, Lee YH, Kang Y, Schiemann WP. TGF-beta stimulates Pyk2 expression as part of an epithelial-mesenchymal transition program required for metastatic outgrowth of breast cancer. Oncogene. 2013;32: 2005-15.

19. Nicolini A, Ferrari P, Fini M, Borsari V, Fallahi P, Antonelli A, et al. Stem cells: their role in breast cancer development and resistance to treatment. Curr Pharm Biotechnol. 2011;12: 196-205.

20. Wang Y, Xu Z, Jiang J, Xu C, Kang J, Xiao L, et al. Endogenous miRNA sponge lincRNA-RoR regulates Oct4, Nanog, and Sox2 in human embryonic stem cell self-renewal. Dev Cell. 2013;25:69-80.

21. Yanamoto S, Yamada S, Takahashi H, Naruse T, Matsushita $\mathrm{Y}$, Ikeda $\mathrm{H}$, et al. Expression of the cancer stem cell markers CD44v6 and ABCG2 in tongue cancer: effect of neoadjuvant chemotherapy on local recurrence. Int J Oncol. 2014;44: 1153-62.

22. Tsai HL, Deng WP, Lai WF, Chiu WT, Yang CB, Tsai YH, et al. Wnts enhance neurotrophin-induced neuronal differentiation in adult bone-marrow-derived mesenchymal stem cells via canonical and noncanonical signaling pathways. PLoS One. 2014;9:e104937.

23. Aguirre A, Rubio ME, Gallo V. Notch and EGFR pathway interaction regulates neural stem cell number and selfrenewal. Nature. 2010;467:323-7.

24. Sirko S, Behrendt G, Johansson PA, Tripathi P, Costa M, Bek $S$, et al. Reactive glia in the injured brain acquire stem cell properties in response to sonic hedgehog. [corrected]. Cell Stem Cell. 2013;12:426-39.

25. Micalizzi DS, Farabaugh SM, Ford HL. Epithelial-mesenchymal transition in cancer: parallels between normal development and tumor progression. J Mammary Gland Biol Neoplasia. 2010;15:117-34. 